\title{
Exosomes and exosomal cargo in urinary extracellular vesicles: novel potential biomarkers for mineralocorticoid-receptor-associated hypertension
}

\author{
Hirotaka Shibata ${ }^{1}$ \\ Received: 22 August 2021 / Accepted: 30 August 2021 / Published online: 1 October 2021 \\ (c) The Japanese Society of Hypertension 2021
}

Hypertension accounts for the largest portion of all cardiovascular deaths in Japan. The number of individuals with hypertension in Japan is estimated to be 43 million, including 31 million individuals with poorly controlled hypertension [1]. The poor control of hypertension may be attributable to patients' poor adherence to treatment, inadequate lifestyle, and "clinical inertia," which includes treatment inertia and diagnostic inertia.

The role of aldosterone in hypertension is best illustrated in primary aldosteronism (PA), which is characterized by aldosterone excess, suppressed plasma renin activity or concentration, and occasionally resistant hypertension. PA is an important cause of endocrine hypertension because of its high prevalence in patients with hypertension as well as its high cardiovascular morbidity compared with essential hypertension [2, 3]. In resistant hypertension, defined as the failure of three or more concomitantly used different classes of antihypertensive drugs to control blood pressure, add-on therapy with a mineralocorticoid receptor (MR) antagonist is frequently effective, and we thereby designate this type of hypertension as "MR-associated hypertension" [4]. MRassociated hypertension is classified into two subtypes: that with high plasma aldosterone levels and that with normal plasma aldosterone levels. The former subtype includes PA and aldosterone-associated hypertension, which show an elevated aldosterone-to-renin ratio but are negative for PA confirmatory tests and obstructive sleep apnea. In contrast, the latter subtype includes obesity, diabetes mellitus, chronic kidney disease, and polycystic ovary syndrome.

Hirotaka Shibata

hiro-405@cb3.so-net.ne.jp

1 Department of Endocrinology, Metabolism, Rheumatology and Nephrology, Faculty of Medicine, Oita University, Yufu 8795593, Japan
The pathogenesis of MR-associated hypertension includes several disease states, such as increased MR levels, increased MR sensitivity, and MR overstimulation by other factors, such as Rac1 [4].

Exosomes and microvesicles are involved in several metabolic processes in tissues and body fluids and are considered to be vehicles for eradicating cell waste products. Exosomes and microvesicles are currently defined as extracellular vesicles of endosomal origin, with a spherical shape and a phospholipid bilayer structure $30-150 \mathrm{~nm}$ in diameter, carrying exosomal markers (cargo) [5]. Recent urinary proteomic studies have identified potential protein biomarkers of renal disease, such as nephrin or podocin, but none of them have been utilized in clinical practice. Urinary exosomes originate from cells lining the nephron lumen and the urinary tract. Plasma exosomes cannot cross the glomerular filtration apparatus; therefore, urinary exosomes originate exclusively from luminal epithelial renal cells. Proteins detected in urinary exosomes may reflect proteins in renal tissues. In research, urinary exosomes are used more than circulating exosomes [6] for the diagnosis of hypertension, probably because most sodium transporters are present on the apical plasma membrane of the kidney epithelium and the release of urinary exosomes to the lumen of the nephron is under hormonal regulation [7, 8] (Fig. 1).

Pendrin, encoded by $S L C 26 A 4$, is selectively present in the $\beta$-intercalated cells and non- $\alpha$, non- $\beta$ intercalated cells of the kidney, mediating $\mathrm{Cl}^{-}$reabsorption in exchange for $\mathrm{HCO}^{-}$secretion. Interestingly, a recent article published by Ochiai-Homma et al. [9] in Hypertension Research showed that, in patients with PA, pendrin abundance in urinary extracellular vesicles (uEVs) is attenuated by therapeutic interventions such as unilateral adrenalectomy or the administration of MR antagonists. In model rats given a continuous infusion of aldosterone, pendrin levels in $\mathrm{uEVs}$, as well as those of epithelial $\mathrm{Na}^{+}$channels and $\mathrm{Na}-\mathrm{Cl}$ cotransporters, were highly correlated with renal abundance. 


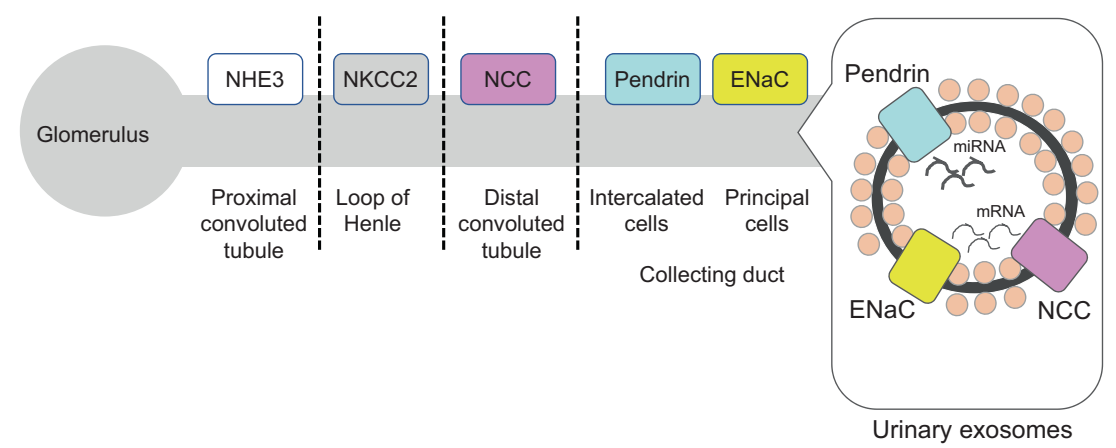

Fig. 1 Urinary exosomes derived from different nephron segments. Urinary exosomes contain specific proteins, mRNA, and microRNA (miRNA) that are associated with mineralocorticoid-receptorassociated hypertension. NHE3 sodium-hydrogen exchanger 3 (encoded by $S L C 9 A 3)$, NKCC2 Na-K-Cl cotransporter (encoded by $S L C 12 A 1$ ), NCC Na-Cl cotransporter (encoded by SLC12A3), Pendrin $\mathrm{Cl}$ - and HCO3- exchanger (encoded by SLC26A4), ENaC epithelial sodium channel (encoded by SCNNI).
In PA patients, pendrin levels in uEVs correlated with the aldosterone-to-renin ratio and were reduced by $49 \%$ from baseline by adrenalectomy or pharmacological MR blockade. Correlation analysis revealed that the magnitude of pendrin reduction after treatment was significantly correlated with the baseline aldosterone-to-renin ratio. Taken together with human and rodent experimental data, these findings indicate that $\mathrm{uEV}$ levels of pendrin and other proteins could be useful biomarkers of aldosterone excess, namely, MR activation in vivo. From a clinical point of view, the discovery of a novel biomarker, uEV pendrin, may be useful for aldosterone excess; however, there are still several limitations. First, we do not know the time lag between the MRmediated activation of pendrin and the detection of exosomes in uEVs. Second, it is crucial to investigate the $\mathrm{uEV}$ pendrin cutoff values in sufficient numbers of PA and healthy control subjects for $\mathrm{uEV}$ pendrin to be used as a biomarker of MR activation. Third, it is important to know which exosomal cargo in uEVs is the most sensitive biomarker of MR transactivation in renal tubular cells.

Among patients who have been diagnosed with "nonPA," there may be a considerable proportion whose hypertension is dependent on MR activation, since the diagnosis of PA is made on the basis of numerical cutoff values of the aldosterone-to-renin ratio. We therefore have proposed a diagnosis of "borderline aldosteronism" in which the plasma aldosterone-to-renin ratio is mildly elevated but PA is not present [10]. MR-associated hypertension includes a wide spectrum of MR activation, such as PA and borderline aldosteronism. It would be unclear to make a diagnosis of MR-associated hypertension because useful clinical biomarkers of MR activation are lacking. Therefore, one way to manage MR-associated hypertension would be to add an MR antagonist to previously used antihypertensive drugs. If blood pressure is effectively reduced, then MR activation is involved in the pathogenesis of hypertension. However, if there are useful and more sensitive biomarkers of MR activation other than hypokalemia and suppressed plasma renin activity, potential candidates for MR-associated hypertension can easily be identified. Among PA patients with aldosterone-producing adenomas, most show spontaneous hypokalemia, whereas more than half of PA patients with bilateral adrenal hyperplasia show normal serum potassium levels. Therefore, hypokalemia is not a sensitive biomarker of PA- or MR-associated hypertension. In contrast, most PA patients show suppressed plasma renin activity in both PA subtypes. However, plasma renin activity may also be suppressed in the case of excessive dietary salt intake as well as genetically low renin essential hypertension. Therefore, low plasma renin may be of limited use as a biomarker of MR-associated hypertension. Since MR-associated hypertension may be one of the reasons for clinical inertia, a good and sensitive biomarker remains to be elucidated. Exosomal markers, including pendrin in uEVs, may be potential good and sensitive biomarkers for MR-associated hypertension. Future research is definitely needed to determine a uEV pendrin cutoff value to be able to use this biomarker in daily clinical practice.

\section{Compliance with ethical standards}

Conflict of interest The author received honoraria from Daiichi Sankyo Co., Limited, and scholarship from Chugai Pharmaceuticals, Limited, and Bayer Pharmaceuticals, Limited.

Publisher's note Springer Nature remains neutral with regard to jurisdictional claims in published maps and institutional affiliations.

\section{References}

1. Umemura S, Arima H, Arima S, Asayama K, Dohi Y, Hirooka Y, et al. The Japanese Society of Hypertension Guidelines for the 
Management of Hypertension (JSH 2019). Hypertens Res. 2019;42:1235-481.

2. Ohno Y, Sone M, Inagaki N, Yamasaki T, Ogawa O, Takeda Y, et al. Prevalence of cardiovascular disease and its risk factors in primary aldosteronism: a multicenter study in Japan. Hypertension. 2018;71:530-7.

3. Satoh M, Maruhashi T, Yoshida Y, Shibata H. Systematic review of the clinical outcomes of mineralocorticoid receptor antagonist treatment versus adrenalectomy in patients with primary aldosteronism. Hypertens Res. 2019;42:817-24.

4. Shibata H, Itoh H. Mineralocorticoid receptor-associated hypertension and its organ damage: clinical relevance for resistant hypertension. Am J Hypertens. 2012;25:514-23.

5. Barros ER, Carvajal CA. Urinary exosomes and their cargo: potential biomarkers for mineralocorticoid arterial hypertension? Front Endocrinol. 2017;8:230 https://doi.org/10.3389/fendo.2017.00230

6. Burrello J, Tetti M, Forestiero V, Biemmi V, Bolis S, Pomatto $\mathrm{MAC}$, et al. Characterization of circulating extracellular vesicle surface antigens in patients with primary aldosteronism. Hypertension. 2021;78:726-37.

7. van der Lubbe N, Jansen PM, Salih M, Fenton RA, van den Meiracker AH, Danser AH, et al. The phosphorylated sodium chloride cotransporter in urinary exosomes is superior to prostasin as a marker for aldosteronism. Hypertension. 2012;60:741-8.

8. Wolley MJ, Wu A, Zu S, Gordon RD, Fenton RA, Stowasser M. In primary aldosteronism, mineralocorticoids influence exosomal sodium-chloride cotransporter abundance. J Am Soc Nephrol. 2017;28:56-63.

9. Ochiai-Homma F, Kuribayashi-Okuma E, Tsurutani Y, Ishizawa $\mathrm{K}$, Fujii W, Odajima K, et al. Characterization of pendrin in urinary extracellular vesicles in a rat model of aldosterone excess and in human primary aldosteronism. Hypertens Res. 2021. https://doi.org/10.1038/s41440-021-00710-5.

10. Tanaka A, Shibata H, Node K. Suspected borderline aldosteronism in hypertension. The next target? J Am Coll Cardiol. 2020;76:759-60. 